\title{
Analysis of Factors Affecting Dental Hygiene Students' Attitudes toward Patient Safety and Performing Confidence
}

\author{
Kyung-Yi Chung ${ }^{\dagger}$ \\ Department of Dental Hygiene, Honam University, Gwangju 62399, Korea
}

The purpose of this study was to investigate the attitudes and performing confidence, and factors influencing patient safety of dental hygiene students. The questionnaire contained items on the subjects' general characteristics, attitudes, and performing confidence in relation to patient safety. The self-reported questionnaire was administered to 287 dental hygiene students in Gwangju and Jeonnam, Korea from September to November 2017. We performed an independent t-test, one-way ANOVA analysis, Pearson's correlation coefficient, and multiple regression analysis using IBM SPSS ver. 21.0 software. As a result of differences in attitude, and performing confidence according to the general characteristics, attitude toward patient safety scored 3.48 out of 5 points, and was more positive in students from 4-year colleges, with high major satisfaction, and in students who answered that they needed patient safety education. Performing confidence for patient safety scored 3.57 out of 5 points, and was statistically significantly higher in 4-year college students, with higher major satisfaction, amongst those with experience in patient safety education, and when patient safety education was needed before clinical practice or employment. There were positive correlations between attitude and performing confidence for patient safety. As a result of analyzing the factors influencing attitude and performing confidence for patient safety, attitudes were higher with higher performing confidence and performing confidence had the highest satisfaction rate of the major and patient safety education experience. Therefore, it is necessary to provide systematic education at universities reflecting the characteristics of dental medical institutions, as well as developing various education contents and education methods.

Key Words: Attitude, Dental hygiene students, Patient safety, Performing confidence

\section{Introduction}

Patient safety is a concept that highlights "patientoriented medicine" for patients who face a threat to their safety, not focusing on medical practitioners ${ }^{1}$. Its purpose is to prevent potential incidents that pose threats to patients such as medical accidents, errors, and mistakes in advance $^{2)}$. Safety accidents that occur to patients at hospitals are an important factor that directly affects not only the quality of the medical service but also the lives of patients, and such accidents can also cause the loss of individual and national medical expenses ${ }^{3)}$.

In 2010, an incident occurred in which a child receiving cancer treatment for leukemia died due to the incorrect injection of the anticancer drug Vincristine into the spinal cord. The incident triggered continuous petitioning for the establishment of a Patient Safety Act. This law was finally enacted in January 2015 and enforced in July 2016 ${ }^{4}$. However, according to the current Patient Safety Act, only hospital-level medical institutions that have more than a certain number of beds are currently obliged to have a patient safety commission and dedicated personnel for the commission. Therefore, there might exist differences in patient safety between general hospitals and some medium-sized and clinic-level medical institutions ${ }^{5}$.

Notably, as of 2016, out of the medical institutions registered in the medical institution information of the Health Insurance Review \& Assessment Service, the 
number of dental medical institutions that were subject to the Act was zero. In addition, only $13.0 \%$ of dental clinics had an awareness of the Patient Safety Act and only $45.5 \%$ of dental clinics with fewer than 200 beds had a patient safety commission. In addition, only $36.4 \%$ had personnel dedicated to patient safety. Regarding experience of patient safety education and the existence of procedures for patient safety, the standard for dental clinics was lower than that for other types of clinic ${ }^{5)}$.

In university general hospitals, dental hospitals, and dental clinics in some regions, $59.4 \%$ of dental hygienists have experience of medical disputes, $24 \%$ of which developed into legal conflicts, according to the result of a previous study ${ }^{6}$. It can be seen from this result that all the members of a dental clinic need to have awareness of the seriousness of patient safety accidents and pay more attention to patient safety for the purpose of preventing medical errors or mistakes.

Dental hygienists are licensed dental medical practitioners who provide diverse dental medical services for the enhancement of dental health and they must play the important role of enhancing patients' safety. It is essential for dental hygienists to have a positive attitude and performance capabilities for the safety of patients, in order to reduce patient safety accidents and improve the quality of dental medical services.

Nevertheless, although dental hygiene students need systematic patient safety training from the college education level in order to perform key dental hygienic skills safely and skillfully after graduation, such training is still insufficient in Korea.

So far, regarding dental patients' safety, studies on the patient safety culture perception of those who work for dental clinics $^{7,8)}$ or dental hygienists ${ }^{9-11)}$ have been conducted. In addition, studies about medical practitioners' attitudes toward patient safety or their performance capabilities have mostly been conducted on registered nurses $^{12,13)}$ or nursing college students ${ }^{2,14,15)}$. However, there have only been a few studies on dental hygiene students' attitudes toward patient safety and their performing confidence in Korea, and so more studies on this subject need to be conducted. This study identifies factors that affect dental hygiene students' attitudes toward patient safety and their performing confidence, to review the need for systematic patient safety education for dental hygiene students at university.

\section{Materials and Methods}

\section{Subjects}

This study was conducted on 2 4 year students of dental hygiene department in four universities located in Gwangju City and Jeollanam-do Province who have experience of clinical training, from September 13 to November 30, 2017.

When the significance level, effect size, statistical power, and number of independent variables were assumed at the $0.05,0.10,0.95$, and 10 levels, respectively, to calculate the sample size in multiple regression analysis using the $\mathrm{G}$ *Power 3.14 program, at least 254 people were needed for the survey. Therefore, the survey was conducted on a total of 287 people. The study was conducted under the approval of the ethics commission of Honam University (1041223-201708-HR-19). The researchers explained the purpose of the study and conducted a self-administered questionnaire survey on those who agreed to participate in the research.

\section{Study tool}

The research tool used for this study was a tool to measure the attitude toward patient safety and performing confidence that was used in the research of Park and Park $^{2)}$. The questionnaire included six questions about the general characteristics of the respondents, 16 questions about their attitudes, and 10 questions about their performing confidence. A 5-point Likert scale was used to measure the respondents' attitudes toward patient safety and their performance confidence. On the scale, a higher score means a more positive attitude toward patient safety and a higher performing confidence. The reliability of the tool used for this study was Cronbach's $\alpha=0.72$ for attitude toward patient safety and Cronbach's $\alpha=0.88$ for performing confidence.

\section{Data analysis}

IBM SPSS Statistics 21.0 for Windows program (IBM 
Co., Armonk, NY, USA) was used for the data analysis. Frequency and percentage were calculated for the general characteristics of the respondents. An independent t-test and one-way ANOVA were used to compare the differences in attitude toward patient safety and their performance confidence according to the general characteristics of the respondents between groups. A Scheffe test was conducted for the post-hoc comparison. The correlation between attitude toward patient safety and performing confidence was analyzed using the Pearson's correlation coefficient. Multiple regression analysis was conducted to identify factors that affect respondents' attitudes toward patient safety and their performing confidence.

\section{Results}

\section{General characteristics of respondents}

The general characteristics of the study respondents are presented in Table 1. Third-year students comprised the largest proportion at $74.9 \%$, while students enrolled in three-year colleges occupied the largest share at $65.2 \%$ and students with a grade point average of 3.0 to 3.4 points occupied the biggest share at $49.5 \%$. Regarding the satisfaction level with their major, $65.2 \%$ of respondents answered "regular," followed by $30.0 \%$ who were "satisfied" and $4.9 \%$ who were "dissatisfied" with their majors. Among the students, $51.2 \%$ had experience of receiving education about patient safety, while the remaining $48.8 \%$ had no such experience. Most of such education programs had been given at their colleges $(86.4 \%)$. In addition, more than half of the respondents, at $61.9 \%$, had no experience of patient safety education through poster display, patient safety events, or videos given at hospitals or colleges. The majority of the respondents $(92.7 \%)$ answered that education about patient safety is needed before clinical training or employment and $86.1 \%$ responded that the reason for the necessity of such education is for the safety of patients.
Table 1. General Characteristics of the Subjects $(n=287)$

\begin{tabular}{llr}
\hline \multicolumn{1}{c}{ Variable } & \multicolumn{1}{c}{ Division } & Frequency \\
\hline Grade level & 2 & $37(12.9)$ \\
& 3 & $215(74.9)$ \\
Education system & 4 & $35(12.2)$ \\
& 3 y & $187(65.2)$ \\
Academic performance & $\geq 4.0$ & $100(34.8)$ \\
& $3.5 \sim 3.9$ & $19(6.6)$ \\
& $3.0 \sim 3.4$ & $61(21.3)$ \\
Major satisfaction & $<3.0$ & $142(49.5)$ \\
& Satisfied & $65(22.6)$ \\
Experience of patient & Somewhat & $86(30.0)$ \\
safety education & Not satisfied & $187(65.2)$ \\
Place of education & No & $14(4.9)$ \\
(n=147) & School & $147(51.2)$ \\
& Dental hospital & $140(48.8)$ \\
Experience of patient & Dental clinic & $127(86.4)$ \\
safety campaign (n=147) & Yes & $3(2.0)$ \\
Need patient safety & Yes & $56(38.1)$ \\
education before clinical & No & $91(61.9)$ \\
practice or employment & & $266(92.7)$ \\
Reason for education & Expansion of dental & $33(12.4)$ \\
(n=266) & hygienist work & \\
& Patient safety & $229(86.1)$ \\
& Self-development & $4(1.5)$ \\
\hline
\end{tabular}

Values are presented as $\mathrm{n}(\%)$.

\section{Attitude toward patient safety and performance confidence according to the general characteristics of respondents}

Table 2 shows the result of comparison for the differences in attitude toward patient safety and performance confidence according to the general characteristics of the study subjects. The average score of attitude toward patient safety was 3.48 out of 5 . The score showed a statistically significant difference according to college course, level of respondents' satisfaction with their major, as well as their awareness of the necessity for patient safety education before clinical training or employment. Those who are enrolled in four-year colleges $(p<0.001)$, who are satisfied with their majors $(p<0.05)$, and who answered that patient safety education is necessary showed a more positive attitude $(p<0.05)$.

The score of performing confidence for patient safety was on average 3.57 out of 5 . The score showed a 
Table 2. Difference of Attitude and Performing Confidence according to the General Characteristics $(n=287)$

\begin{tabular}{|c|c|c|c|}
\hline \multicolumn{2}{|l|}{ Variable } & \multirow{2}{*}{$\begin{array}{c}\text { Attitude } \\
3.42 \pm 0.30\end{array}$} & \multirow{2}{*}{$\begin{array}{c}\text { Performing confidence } \\
3.52 \pm 0.53\end{array}$} \\
\hline Education system & $3 y$ & & \\
\hline & $4 \mathrm{y}$ & $3.60 \pm 0.36$ & $3.66 \pm 0.46$ \\
\hline & $\mathrm{t}(\mathrm{p})$ & $-4.324(<0.001)$ & $-2.211(0.028)$ \\
\hline \multirow[t]{5}{*}{ Academic performance } & $\geq 4.0$ & $3.56 \pm 0.34$ & $3.64 \pm 0.59$ \\
\hline & $3.5 \sim 3.9$ & $3.52 \pm 0.35$ & $3.57 \pm 0.47$ \\
\hline & $3.0 \sim 3.4$ & $3.49 \pm 0.33$ & $3.55 \pm 0.52$ \\
\hline & $<3.0$ & $3.41 \pm 0.33$ & $3.59 \pm 0.50$ \\
\hline & $\mathrm{F}(\mathrm{p})$ & $1.645(0.179)$ & $0.242(0.867)$ \\
\hline \multirow[t]{4}{*}{ Major satisfaction } & Satisfied & $3.53 \pm 0.32^{\mathrm{a}}$ & $3.76 \pm 0.50^{\mathrm{a}}$ \\
\hline & Somewhat & $3.48 \pm 0.33^{\mathrm{a}}$ & $3.52 \pm 0.48^{\mathrm{a}}$ \\
\hline & Not satisfied & $3.27 \pm 0.37^{\mathrm{b}}$ & $3.12 \pm 0.48^{\mathrm{b}}$ \\
\hline & $\mathrm{F}(\mathrm{p})$ & $3.637(0.028)$ & $13.851(<0.001)$ \\
\hline \multirow[t]{3}{*}{ Experience of patient safety education } & Yes & $3.47 \pm 0.34$ & $3.64 \pm 0.51$ \\
\hline & No & $3.50 \pm 0.33$ & $3.49 \pm 0.49$ \\
\hline & $\mathrm{t}(\mathrm{p})$ & $-0.753(0.452)$ & $2.499(0.013)$ \\
\hline \multirow[t]{3}{*}{ Need patient safety education before clinical practice or employment } & Yes & $3.49 \pm 0.34$ & $3.59 \pm 0.51$ \\
\hline & No & $3.34 \pm 0.26$ & $3.33 \pm 0.39$ \\
\hline & $\mathrm{t}(\mathrm{p})$ & $2.096(0.037)$ & $2.236(0.026)$ \\
\hline Total & & $3.48 \pm 0.33$ & $3.57 \pm 0.51$ \\
\hline
\end{tabular}

Values are presented as mean \pm standard deviation.

${ }^{\mathrm{a}, \mathrm{b}, \mathrm{c}}$ The same letter indicates no significant difference by Scheffe test at $\alpha=0.05$.

statistically significant difference according to the college course, level of respondents' satisfaction with their majors, experience of patient safety education, and their awareness of the necessity for patient safety education before clinical training or employment. Those enrolled in four-year colleges $(\mathrm{p}<0.05)$, who have a high level of satisfaction with their majors $(\mathrm{p}<0.001)$, who have experience of patient safety education $(p<0.05)$, and who answered that patient safety education is necessary $(\mathrm{p}<0.05)$ demonstrated performing confidence at a statistically significant higher level.

\section{Correlation between attitudes toward patient safety and performing confidence}

The correlation between respondents' attitudes toward patient safety and their performing confidence is presented in Table 3. The attitude toward patient safety and performing confidence $(\mathrm{r}=0.357, \mathrm{p}<0.001)$ showed a statistically significant positive correlation.
Table 3. Correlation between Attitude and Performing Confidence on Patients on Patient Safety

\begin{tabular}{lcc}
\hline \multicolumn{1}{c}{ Variable } & Attitude & $\begin{array}{c}\text { Performing } \\
\text { confidence }\end{array}$ \\
\hline $\begin{array}{l}\text { Attitude } \\
\text { Performing confidence }\end{array}$ & $\begin{array}{c}1 \\
0.357^{* * *}\end{array}$ & 1 \\
\hline
\end{tabular}

$* * * \mathrm{p}<0.001$.

\section{Factors that affect attitudes toward patient safety and performing confidence}

A multiple regression analysis was conducted to identify factors that affect respondents' attitudes toward patient safety and their performing confidence and the result is presented in Table 4. It was set at an input method, in order to analyze the influence of independent variables that were considered in the regression analysis. All the fitted models were statistically significant and the considered independent variables showed $17.9 \%$ explanatory power for attitude toward patient safety and 19.8\% explanatory power for performing confidence. Attitude toward patient safety has a statistically significant correlation with the educational system and performing confidence among the independent variables. Those who 
Table 4. Influencing Factors on Attitude and Performing Confidence on Patients on Patient Safety

\begin{tabular}{|c|c|c|c|c|c|c|c|c|}
\hline & Variable & $\mathrm{B}$ & SE & $\beta$ & $\mathrm{t}$ & p-value & $\mathrm{F}(\mathrm{p})$ & Adjusted $\mathrm{R}^{2}$ \\
\hline \multirow[t]{2}{*}{ Attitude } & Education system (4 y) & 0.146 & 0.039 & 0.209 & 3.753 & $<0.001$ & $7.930(<0.001)$ & 0.179 \\
\hline & Performing confidence & 0.225 & 0.038 & 0.341 & 5.967 & $<0.001$ & & \\
\hline \multirow[t]{4}{*}{$\begin{array}{l}\text { Performing } \\
\text { confidence }\end{array}$} & $\begin{array}{l}\text { Major satisfaction } \\
\text { (satisfied) }\end{array}$ & 0.229 & 0.061 & 0.208 & 3.742 & $<0.001$ & $8.851(<0.001)$ & 0.198 \\
\hline & $\begin{array}{l}\text { Major satisfaction } \\
\text { (not satisfied) }\end{array}$ & -0.265 & 0.128 & -0.113 & -2.079 & 0.039 & & \\
\hline & $\begin{array}{l}\text { Experience of patient } \\
\text { safety education (yes) }\end{array}$ & 0.149 & 0.055 & 0.147 & 2.720 & 0.007 & & \\
\hline & Attitude & 0.506 & 0.085 & 0.334 & 5.967 & $<0.001$ & & \\
\hline
\end{tabular}

are enrolled in four-year colleges $(\mathrm{B}=0.146)$ and who have higher performing confidence $(\mathrm{B}=0.225)$ showed a more positive attitude toward patient safety. Performing confidence for patient safety showed a statistically significant correlation with the level of satisfaction with their major, experience of patient safety education, and attitude toward patient safety, out of the independent variables. Those who were more satisfied with their majors $(B=0.229)$, who had more experience of patient safety education $(B=0.149)$, and who had a more positive attitude toward patient safety $(B=0.506)$ showed greater performing confidence for patient safety.

\section{Discussion}

The number of patients who use dental clinics has risen in accordance with continuous advancements of medical technologies. The number of medical lawsuits and disputes over medical accidents at dental clinics has also increased $^{6)}$ as the medical environment has become more complicated. Under such circumstances, people are becoming more interested in patient safety and the quality of medical service ${ }^{16)}$. This study identifies factors that affect the attitude toward patient safety and performing confidence of dental hygiene major students who will play the key role for patient safety as dental hygienists in the future, to review the necessity of systematic patient safety education for dental hygiene students at colleges.

In order to examine dental hygiene students' attitudes toward patient safety, they were asked 16 questions about their awareness of general medical errors and response measures to take when medical errors occur. The total attitude score was 3.48 points out of 5 . When the score was compared with nursing major students' attitudes toward patient safety, the score of dental hygiene students was slightly lower than the 3.77 points of nursing students ${ }^{2)}$ and another study that recorded 3.68 points for nursing students ${ }^{14)}$. This difference might have been due to the difference in the surveying tool used by the researchers. It seems that basic patient safety education is highlighted more in college curriculums for nursing majors, as safety accidents committed by nurses have a more direct influence on patients' lives.

Among the questions about attitude toward patients' safety, the score for the question "occurrence of a medical error at healthcare and medical sites is inevitable" was 3.01; the score for the question "skilled medical practitioners do not commit medical errors that harm patients" was 2.92; the score for the question "the system of reporting medical errors itself does not reduce future occurrence of medical errors" was 2.95; and the score for the question "an effective response to medical errors is to put focus on related medical practitioners" was 2.86, which were the lowest scores.

Case studies conducted by healthcare and medicalrelated departments of foreign colleges have reported that a curriculum structured with immediate feedback and evaluation using diverse educational methods such as role-playing, simulation, small-group discussions, and problem-solving is the most effective method to reinforce students' attitudes toward patient safety ${ }^{17)}$. They have also reported that the score of students' attitudes toward patient safety was increased by educating them with a curriculum that reflects patient safety ${ }^{18)}$. Therefore, it is thought that 
colleges need to actively review the need for a patient safety education curriculum that utilizes diverse educational methods rather than simply focusing on the role of dental hygienists in relation to dental hygiene clinical performance capabilities, so that students can form a desirable attitude toward patient safety.

As for students' performing confidence regarding patient safety, students were asked 10 questions about international patient safety targets and ways of responding to the occurrence of patient safety accidents that students might experience; the average score of their answers was 3.57 out of 5 . When college students majoring in nursing were surveyed using the same surveying tool, they recorded an average score of 3.85 , which indicates that the level of dental hygiene students' performing confidence is generally lower.

The score recorded by dental hygiene students was the highest for the question "polluted garbage is separated into pollutants and extracted tissues for waste discharge, for the purpose of preventing infection" at 4.04 and for the question "I know the proper method of hand hygiene and perform it" at 3.98. The score recorded by nursing students ${ }^{2)}$ was the highest for the question "Wheels are always fixed when moving patients in wheelchairs" at 4.43 and for the question "I make good use of the bed rail and explain the relevant safety" at 4.36 , indicating the difference in the performing confidence between dental hygiene students and nursing students. In short, dental hygiene students have higher performing confidence regarding the reduction of infection in healthcare and medical practice, while nursing students have higher performing confidence for the reduction of risk of patients' fall. This difference seems to have been caused by differences in the educational content frequently provided to students in their clinical training and highlighted in the curriculum. On the other hand, the performing confidence for response to the occurrence of medical errors was low for both groups, indicating the need to enhance such performing confidence by providing intensive education to students on how to respond to medical errors, as they have a chance of facing medical errors while engaging in clinical training.

Performing confidence according to dental hygiene students' general characteristics was analyzed and students who are more satisfied with their majors and who have experience of patient safety education showed a significantly higher level of performing confidence. As such, students' experience of patient safety education and satisfaction with their majors were found to be factors that affect their performing confidence. This result is thought to have been possible as students who are highly satisfied with their majors and have experience of patient safety education have a higher level of understanding of the content of education and a more appropriate attitude toward patient safety is formed in them, leading to the higher level of performing confidence.

Notably, only $51.2 \%$ of dental hygiene students have experience of receiving patient safety education, demonstrating that patient safety education is very lacking for them. In addition, the survey result on the experience of patient safety education among those who work at clinic-level medical institutions found that those who work at dental clinics have less experience of receiving patient safety education ${ }^{5)}$. On the contrary, although the study subjects and researchers were different for a study on nursing students, the percentage of nursing major students who have received patient safety education has increased continuously from $56.3 \%$ in $2014^{2)}$, to $88.8 \%$ in $2015^{14)}$, and then to $79.4 \%$ in $2017^{15)}$. Another study that reviewed the method and content of patient safety education included in the curriculum for healthcare and medical-related departments of colleges in and out of the country demonstrated that such education programs reinforce students' knowledge, attitudes, and techniques in the fields of nursing, pharmacy, and medicine, but no such research has been conducted on dental college students ${ }^{4)}$. There could be a difference in patient safety awareness between dental clinics and other medical institutions, due to the difference in the frequency of patient safety accident occurrence, the environment of the clinics, and the duties of workers. However, as patient safety accidents occur suddenly and unexpectedly and their occurrence is directly related with patients' lives, colleges and clinical training sites should pay more attention to patient safety and implement more practical and detailed patient safety education. 
The correlation between dental hygiene students' attitudes toward patient safety and their performing confidence was analyzed and a statistically significant correlation was found between the two. This was similar to the result drawn by Huh and Kang ${ }^{14)}$, as well as Jeong et al. ${ }^{9)}$. Factors that affect it were analyzed to ascertain that performing confidence for patient safety is higher when the attitude toward patient safety is greater, while greater performing confidence forms a more desirable attitude toward patient safety. Therefore, in order to enhance students' performing confidence for patient safety, they should first receive education regarding the basic concept of patient safety followed by the importance of patient safety, to form a desirable attitude toward patient safety.

This study is meaningful in that it examined the level of dental hygiene students' attitudes toward patient safety and their performing confidence and suggested factors that affect attitudes and confidence, thereby proving the need for systematic patient safety education for college students majoring in dental hygiene. However, it also has a limitation in generalizing the result, as the survey was conducted on dental hygiene students from a limited region. In addition, it has another limitation that some results were compared with the results of nursing students, because there currently exist only a few studies on dental hygiene students. More studies on dental hygiene students from more regions, as well as on the development of a detailed educational curriculum and method and comparison of the result of their application will have to be conducted in the future.

\section{References}

1. Lee JH, Lee SI: The concept and general approach. Qual Improv Health Care 15: 9-18, 2009.

2. Park J, Park M: Knowledge, attitude, and confidence on patient safety of undergraduate nursing students. J Korean Acad Soc Nurs Educ 20: 5-14, 2014. https://doi.org/10.5977/jkasne.2014.20.1.5

3. Kim MR: Concept analysis of patient safety. J Korean Acad Nurs 41: 1-8, 2011. https://doi.org/10.4040/jkan.2011.41.1.1

4. Chun HR, Shin EH: Review on patient safety education for undergraduate/pre-registration curricula in health professions.
Korean Public Health Res 42: 45-58, 2016.

5. Seo JH: Patient safety in small or medium hospitals and clinics: current status. Health Welfare Policy Forum 242: 84-94, 2016.

6. Yang EM, Park SJ, Kim HJ: The experience of medical conflict and the educational needs of dental hygienists. J Dent Hyg Sci 15: 361-368, 2015. https://doi.org/10.17135/jdhs.2015.15.3.361

7. Kim SK, Choi JW, Lee SG, Cho YD, Han EA, Kim TH: The association between patient safety culture perceptions and patient safety management activities in dental hospital workers. J Korean Soc Dent Hyg 16: 1033-1045, 2016. https://doi.org/10.13065/jksdh.2016.16.06.1033

8. Shin HS: Dental culture for patient safety. J Korean Acad Dent Adm 1: 17-29, 2013.

9. Jeong JE, Hwang DH, Yun HK: An analysis on the dental hygienists' perception of patient safety culture at dental clinics. Korean Soc Health Serv Manage 7: 81-92, 2013. https://doi.org/10.12811/kshsm.2013.7.4.081

10. Lee DJ, Han SJ: Influence of perceived patient safety culture on patient safety management activity in the dental hygienists. J Korean Soc Dent Hyg 16: 863-877, 2016. https://doi.org/10.13065/jksdh.2016.16.06.863

11. Choi EM, Noh HJ, Chung WG, Mun SJ: Perception of infection control activities and patient safety culture among dental hygienists. J Korean Soc Dent Hyg 17: 769-777, 2017. https://doi.org/10.13065/jksdh.2017.17.05.769

12. Choi JH, Lee KM, Lee MA: Relationship between hospital nurses' perceived patient safety culture and their safety care activities. J Korean Acad Fundam Nurs 17: 64-72, 2010.

13. Nam MH, Lim JH: The influences of the awareness of patient safety culture on safety care activities among nurse in small-medium sized general hospitals. J Digit Converg 11: 349-359, 2013.

14. Huh SS, Kang HY: The attitude of patient safety and patient safety management activity in nursing students. J Korea Acad-Ind Coop Soc 16: 5458-5467, 2015. https://doi.org/10.5762/KAIS.2015.16.8.5458

15. Jeong HS, Kong JH, Jeon MY: Factors influencing confidence in patient safety management in nursing students. J Korea Converg Soc 8: 121-130, 2017. https://doi.org/10.15207/JKCS.2017.8.6.121

16. Choi SH, Lee H: Factors affecting nursing students' practice 
of patient safety management in clinical practicum. J Korean Acad Nurs Adm 21: 184-192, 2015.

https://doi.org/10.11111/jkana.2015.21.2.184

17. Karl KA, O'Leary-Kelly AM, Martocchio JJ: The impact of feedback and self-efficacy on performance in training. J Org Behav 14: 379-394, 1993. https://doi.org/10.1002/job.4030140409

18. Madigosky WS, Headrick LA, Nelson K, Cox KR, Anderson $\mathrm{T}$ : Changing and sustaining medical students' knowledge, skills, and attitudes about patient safety and medical fallibility. Acad Med 81: 94-101, 2006. 